\title{
Comparative kinome analysis to identify putative colon tumor biomarkers
}

\author{
Ewa E. Hennig • Michal Mikula • Tymon Rubel • \\ Michal Dadlez $\cdot$ Jerzy Ostrowski
}

Received: 30 June 2011 /Revised: 22 October 2011 / Accepted: 28 October 2011 /Published online: 18 November 2011

(C) The Author(s) 2011. This article is published with open access at Springerlink.com

\begin{abstract}
Kinase domains are the type of protein domain most commonly found in genes associated with tumorigenesis. Because of this, the human kinome (the protein kinase component of the genome) represents a promising source of cancer biomarkers and potential targets for novel anticancer therapies. Alterations in the human colon kinome during the progression from normal colon $(\mathrm{NC})$ through adenoma (AD) to adenocarcinoma (AC) were investigated using integrated transcriptomic and proteomic datasets. Two hundred thirty kinase genes and 42 kinase proteins
\end{abstract}

Electronic supplementary material The online version of this article (doi:10.1007/s00109-011-0831-6) contains supplementary material, which is available to authorized users.

E. E. Hennig $\cdot$ J. Ostrowski

Department of Gastroenterology and Hepatology, Medical Center for Postgraduate Education,

Warsaw, Poland

E. E. Hennig $\cdot$ M. Mikula $\cdot$ J. Ostrowski

Department of Oncological Genetics, The Maria SklodowskaCurie Memorial Cancer Center and Institute of Oncology,

Warsaw, Poland

T. Rubel

Institute of Radioelectronics, Warsaw University of Technology,

Warsaw, Poland

\section{Dadlez}

Institute of Biochemistry and Biophysics,

Polish Academy of Sciences,

Warsaw, Poland

J. Ostrowski $(\bowtie)$

Cancer Center Institute,

Roentgena 5,

02-781 Warsaw, Poland

e-mail: jostrow@warman.com.pl showed differential expression patterns (fold change $\geq 1.5$ ) in at least one tissue pair-wise comparison (AD vs. NC, AC vs. NC, and/or AC vs. AD). Kinases that exhibited similar trends in expression at both the mRNA and protein levels were further analyzed in individual samples of $\mathrm{NC}(n=20)$, $\mathrm{AD}(n=39)$, and $\mathrm{AC}(n=24)$ by quantitative reverse transcriptase PCR. Individual samples of NC and tumor tissue were distinguishable based on the mRNA levels of a set of 20 kinases. Altered expression of several of these kinases, including chaperone activity of bc 1 complex-like $(\mathrm{CABC} 1)$ kinase, bromodomain adjacent to zinc finger domain protein 1B (BAZ1B) kinase, calcium/calmodulindependent protein kinase type II subunit delta (CAMK2D), serine/threonine-protein kinase 24 (STK24), vacciniarelated kinase 3 (VRK3), and TAO kinase 3 (TAOK3), has not been previously reported in tumor tissue. These findings may have diagnostic potential and may lead to the development of novel targeted therapeutic interventions for colorectal cancer.

Keywords Protein kinase - Colorectal cancer . Transcriptomics $\cdot$ Proteomics $\cdot$ TAOK3

\section{Introduction}

Protein phosphorylation is a central element of numerous cell signaling pathways, including those that control cell growth, differentiation, apoptosis, and invasion. The relevance of phosphorylation is underscored by the fact that the protein kinase (PK) complement of the genome (the socalled kinome) comprises about $2 \%$ of all human genes, encoding 518 distinct tyrosine or serine/threonine PKs which are involved in the phosphorylation of over one third of all intracellular proteins [1]. 
Aberrant function of PKs has been implicated in malignant transformation and tumor development [2]. A global survey of somatic mutations in a diverse set of human cancer genomes showed that the PK domain is the most commonly represented domain in so-called cancer genes, with the strongest evidence of overrepresentation compared to what would be expected by chance [3]. Mutations in PKs may be involved in the development of a significant proportion of cancers, including colorectal cancer (CRC) [4].

Preliminary evaluation of transcriptomic datasets from a microarray study of oncogenic signaling in CRC [5] revealed that probe sets which represented $\mathrm{PK}$ genes have the potential to distinguished normal colon (NC) epithelium from adenomas (ADs) and adenocarcinomas (ACs) (not shown). Therefore, in the present study, combined transcriptomic and proteomic datasets from our integrated microarray- and mass spectrometry (MS)-based study [6] were mined for PKs whose expression concordantly changed at both the transcriptional and the protein levels during the progression from NC through AD to AC. To our knowledge, a comprehensive approach such as the one taken here for analyzing the tumor kinome has not been reported previously.

\section{Materials and methods}

PK probe sets and peptides

The identifiers (IDs) of the probe sets (Affymetrix) and proteins (Swiss-Prot) were used to select the respective PKs from our previously derived transcriptomic and proteomic datasets established on pooled colonic tissue samples [6]. The putative PKs were searched using the term "protein kinase" to browse the Gene Ontology (GO) database, and the selection was further verified based on data available in the following databases: UniProt (http:/www.uniprot.org/), GeneCards (http://www.genecards.org/), Human Protein Reference Database (http://www.hprd.org/), Bioinformatic Harvester (http://www.harvester.fzk.de), PhosphoSitePlus (http://www.posphosite.org/), Pfam (http://www.pfam. sanger.ac.uk/), Brenda (http://www.brenda-enzyme.org/), and the kinome database of Manning et al. [1].

Functional annotation and biological pathway analysis

The gene and protein lists were assessed for known biological interactions and involvement in canonical pathways using Ingenuity Pathway Analysis (IPA; Ingenuity Systems, Mountain View, CA, http://www.ingenuity.com). The gene/protein IDs were imported into IPA for core analysis evaluation. The Fisher's exact test with the
Benjamini-Hochberg correction for multiple hypotheses was used to determine the probability that the association between the dataset and the biological function or the canonical pathway was due to random chance alone. A $p$ value of less than 0.01 was considered statistically significant. The ratio of the number of genes/proteins in the dataset that mapped to the pathway and the total number of molecules that made up the pathway provided an estimate of the extent of pathway involvement.

Quantitative reverse transcriptase PCR

RNA concentrations were determined in 83 colonic tissue samples using quantitative reverse transcriptase PCR (qRTPCR), as described previously [6]. The clinical characteristics of the patients and the histopathological characteristics of the analyzed tissue samples are presented in Table S1. The sequences of all primers are listed in Table S2. Differences were evaluated using the MannWhitney $U$ test in GraphPad Prism 5 (GraphPad Software, Inc., CA, USA). A $p$ value of less than 0.05 was considered statistically significant.

\section{Results}

Identification of PKs using combined transcriptomic and proteomic datasets

To select sets of PK genes and proteins, combined transcriptomic and proteomic datasets previously acquired for pooled $\mathrm{NC}, \mathrm{AD}$, and $\mathrm{AC}$ tissues in our integrated microarray- and MS-based study [6] were reevaluated. From the 24,740 probe sets that remained after filtering according to the GCRMA+LVS algorithm, 792 probe sets were identified corresponding to $410 \mathrm{PK}$ genes (Table S3). Of these, 308 (75\%) and 69 (17\%) encoded serine/ threonine kinases (STKs) and tyrosine kinases (TKs), respectively. Among 3,886 distinct proteins (identified by at least two peptides), 90 were confirmed as PKs; 63 (70\%) and $24(27 \%)$ were STKs and TKs, respectively. In total, $411 \mathrm{PK}$ transcripts and/or PK proteins were identified (see Table S4 for the full list), with $89 \mathrm{PKs}$ common to both datasets.

Comparative analysis of PK gene expression and protein levels

The first component of principal component analysis (PCA) revealed that $\mathrm{PK}$ mRNA levels distinguished $\mathrm{NC}$ and neoplastic tissues (Fig. 1a), whereas protein levels distinguished $\mathrm{AD}$ from $\mathrm{NC}$ and $\mathrm{AC}$ (Fig. 1b). These results suggested that the changes in $\mathrm{PK}$ mRNA and protein 
Fig. 1 PCA. The first two principal components were determined based on the expression of $792 \mathrm{PK}$ probe sets from the mRNA microarray survey (a) and $90 \mathrm{PKs}$ from the proteomic analysis (b). $A C$ adenocarcinoma, $A D$ adenoma, $N C$ normal colon a

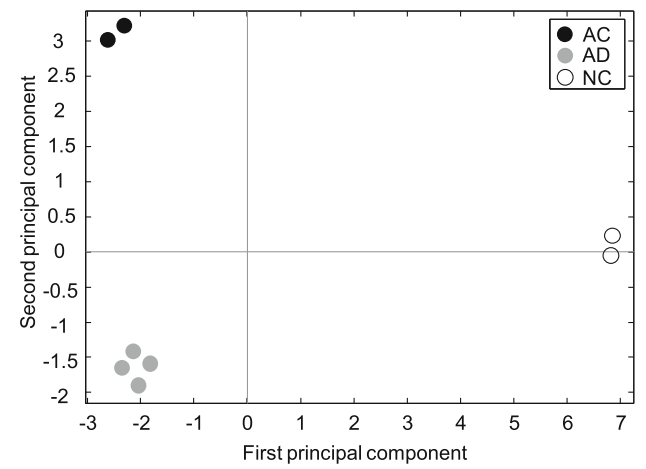

b

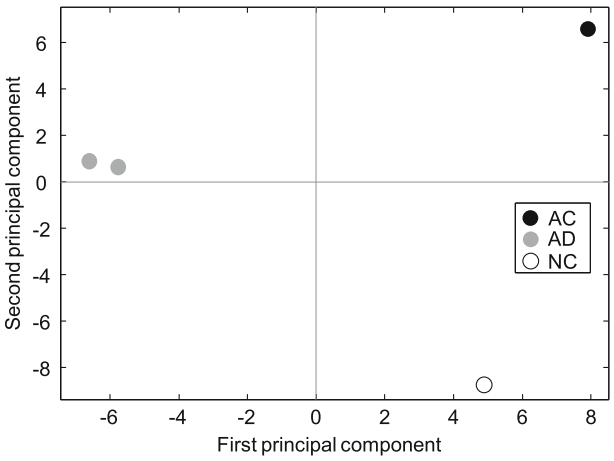

expression during CRC progression are to some extent quantitatively different.

Pair-wise comparisons revealed that 164, 81, and 199 genes were differentially expressed ( $\geq 1.5$-fold) in $\mathrm{AD}$ vs. $\mathrm{NC}, \mathrm{AC}$ vs. AD, and $\mathrm{AC}$ vs. NC, respectively (Fig. S1a), as calculated by geometric mean ratio values for probe sets representing a single PK gene. However, a substantially smaller number of PKs were differentially expressed at the protein level, with 23,22 , and 26 proteins exhibiting different expression levels in $\mathrm{AD}$ vs. $\mathrm{NC}, \mathrm{AC}$ vs. $\mathrm{AD}$, and AC vs. NC, respectively (Fig. S1b). In total, the levels of 230 transcripts and 42 proteins were different in at least one pair-wise comparison (Table S5), which most likely reflects the differences in the sensitivity of microarray and MS techniques.

Comparison of expression trends and functional annotation of differentially expressed PKs

Based on pair-wise comparisons and using a threshold of fold change $(\mathrm{FC}) \geq 1.5$, PK levels were assigned to one of five expression trends, as shown in Table 1. Trend 3 was the most prevalent among the differentiating PK transcripts and proteins; however, strikingly, trend 5 was almost four times more frequent among proteins than genes.

Functional annotation of the differentially expressed PK genes and proteins and assignment to Ingenuity canonical signaling pathways was carried out using IPA software (Tables S6 and S7). Of note, 26 of the 36 process categories that were significantly associated with the PK proteins were also among the top 40 categories for the PK genes. Furthermore, the top five canonical pathways identified for the protein dataset were among the top 23 pathways identified for the gene dataset. Thus, while there were differences observed in the expression trends for PK mRNAs and proteins during CRC progression, these results suggested that the changes may be functionally related and might affect a limited number of signaling pathways.
Functional relationships among differentially expressed kinases

To identify putative kinase markers of CRC development, we focused on those kinases that exhibited changes at both the mRNA and protein levels. Of 230 differentially expressed PK genes and 42 proteins, 24 exhibited changes at the transcriptional and protein levels. Of these, 20 were consistent in the direction of change (Table 2). It should be noted, however, that despite concordance in the direction of the change, the magnitude of the change differed considerably at the mRNA and protein levels for some proteins/ genes.

The most significant functional annotations of the 20 PKs that exhibited concordant changes at the transcriptional and protein levels are listed in Table S8. These PKs were associated with a wide spectrum of developmental processes, as well as survival, tissue and cell morphology, and cell movement. Strikingly, several functional associations were related to vascular and nervous system development and function.

As defined by IPA, the 20 kinases clustered with other molecules stored in the Ingenuity Knowledge Base into two interaction networks that were algorithmically generated based on connectivity. The first network included 14 kinases (score 37, $p$ value $<10^{-37}$ ) and was functionally associated with cardiovascular system development and function, embryonic development, and tissue development. The second network contained the remaining six kinases (score 13) and was associated with cell cycle, cell death, and infection mechanisms. Merging the two networks into one that included all $20 \mathrm{PKs}$ resulted in a network with the tumor suppressor TP53 as the hub (Fig. 2 and Table S9).

\section{Potential markers of CRC progression}

The expression of the selected 20 PKs was finally evaluated by qRT-PCR in 83 tissue samples (Fig. 3 and Table S10). Overall, the results of the qRT-PCR were in agreement with the results of the large-scale analysis of the transcriptomic 
Table 1 Trends in PK expression during progression from normal colonic mucosa to adenoma and from adenoma to colorectal adenocarcinoma

\begin{tabular}{|c|c|c|c|}
\hline \multirow{2}{*}{\multicolumn{2}{|c|}{$\begin{array}{l}\text { Difference in fold-change in } \\
\text { two pair-wise comparisons }{ }^{\mathrm{a}} \\
\text { Graphical depiction of trends } \\
\text { of changes }\end{array}$}} & \multicolumn{2}{|c|}{$\begin{array}{c}\text { Number (percent) of differentiating } \\
\text { PK genes or proteins }\end{array}$} \\
\hline & & $\begin{array}{l}\text { Transcriptome (genes) } \\
\qquad n=230\end{array}$ & $\begin{array}{c}\text { Proteome (proteins) } \\
n=42\end{array}$ \\
\hline 1 & & $26(11.3)$ & 7 (16.7) \\
\hline 2 & & $27(11.7)$ & $0(0)$ \\
\hline 3 & & $123(53.5)$ & $13(31.0)$ \\
\hline 4 & & $40(17.4)$ & $12(28.6)$ \\
\hline 5 & (NC) & $14(6.1)$ & $10(23.8)$ \\
\hline
\end{tabular}

$N C$ normal colon, $A D$ adenoma, $A C$ adenocarcinoma

${ }^{\text {a }}$ Pair-wise comparisons were between $\mathrm{AD}$ and $\mathrm{NC}$ and between $\mathrm{AC}$ and $\mathrm{AD}$. Differences in fold-change between groups of $\geq 1.5$ are represented by the solid arrows; changes $<1.5$ are represented by the dashed arrows

${ }^{\mathrm{b}}$ Trend 1 represents PKs that exhibited significant change only in AC vs. NC comparison. Trend 2 exhibits PKs progressively up- or downregulated from $\mathrm{NC}$ to $\mathrm{AD}$ and $\mathrm{AD}$ to $\mathrm{AC}$. Trend 3 represents changes in expression upon progression from $\mathrm{NC}$ to $\mathrm{AD}$ with no corresponding changes in the transition from $\mathrm{AD}$ to $\mathrm{AC}$. Changes associated with progression to malignancy (AC vs. $\mathrm{AD}$ ) with no corresponding changes in the early stages of the neoplastic process were classified as trend 4 . Trend 5 represents regressive changes (opposite direction in each comparison)

and proteomic datasets, with the exception of SFRS protein kinase 2 (SRPK2), fyn-related kinase (FRK), and cAMPdependent protein kinase catalytic beta (PRKACB). For these three kinases, the direction of the change in gene expression by qRT-PCR was different from that shown by transcriptomic/proteomic profiling in at least one pair-wise comparison. Moreover, the expression of MST4 kinase correlated with AC staging (Spearman= $0.537 ; p=0.007)$ and significantly differentiated B1 vs. B2 $(p=0.0475)$ and $\mathrm{B} 1$ vs. $\mathrm{C} 2(p=0.0061)$ stages of $\mathrm{AC}$ (Fig. S2).

When subjected to hierarchical clustering, the qRT-PCR expression patterns clearly distinguished NC samples (with the exception of one sample) from all other neoplastic tissues (Fig. 4). The NC samples consisted of specimens derived from normal epithelium adjacent $(>5 \mathrm{~cm})$ to adenocarcinoma (NC-A), as well as tissue from healthy individuals obtained during colonoscopy (NC-D). Interestingly, the expression profiles distinguished five of six $\mathrm{NC}$ $\mathrm{D}$ tissue samples from NC-A. One possibility for this distinction is that the cancer tissue might influence gene expression within the adjacent normal epithelium. It is also noteworthy that the expression of TAO kinase 3 (TAOK3) was significantly different $(p<0.001)$ between these two types of NC (Fig. 3). This observation may have diagnostic implications, since $T A O K 3$ expression appears to be suppressed within colonic epithelium in the vicinity of carcinoma.

To determine if differences measured on the mRNA and protein levels correspond with kinases activity, kinome profiling was performed using PepChip Kinomics peptide array (Electronic supplementary materials). Eight of the selected $20 \mathrm{PKs}$ were represented by their substrates on the array, and for seven of them, the 
Table 2 PKs exhibiting concordant changes in expression at the transcriptional and protein levels during CRC progression

\begin{tabular}{|c|c|c|c|c|c|c|c|c|}
\hline \multirow[t]{2}{*}{ Symbol } & \multirow[t]{2}{*}{ Swiss ID } & \multirow[t]{2}{*}{ Name } & \multicolumn{3}{|c|}{ Transcriptomics } & \multicolumn{3}{|c|}{ Proteomics } \\
\hline & & & $\begin{array}{l}\mathrm{AD} / \\
\mathrm{NC}\end{array}$ & $\begin{array}{l}\mathrm{AC} / \\
\mathrm{AD}\end{array}$ & $\begin{array}{l}\mathrm{AC} / \\
\mathrm{NC}\end{array}$ & $\begin{array}{l}\mathrm{AD} / \\
\mathrm{NC}\end{array}$ & $\begin{array}{l}\mathrm{AC} / \\
\mathrm{AD}\end{array}$ & $\begin{array}{l}\mathrm{AC} / \\
\mathrm{NC}\end{array}$ \\
\hline $\mathrm{CABC} 1$ & Q8NI60 & Chaperone activity of bc1 complex-like & 2.84 & 0.63 & 1.78 & $3.13 *$ & $0.40^{*}$ & 1.25 \\
\hline EPHB2 & P29323 & EPH receptor B2 & 5.80 & 0.62 & 3.61 & $1.81 *$ & $0.62 *$ & 1.13 \\
\hline EPHB3 & P54753 & EPH receptor B3 & 23.72 & 0.44 & 10.37 & $2.92 *$ & $0.67 *$ & $1.95 *$ \\
\hline EPHB4 & P54760 & EPH receptor B4 & 3.32 & 0.73 & 2.42 & $2.35 *$ & $0.58^{*}$ & 1.38 \\
\hline SRPK1 & Q96SB4 & SFRS protein kinase 1 & 2.23 & 0.93 & 2.07 & 1.91 & 1.04 & 1.98 \\
\hline SRPK2 & P78362 & SFRS protein kinase 2 & 2.15 & 1.05 & 2.25 & 1.06 & 1.65 & 1.75 \\
\hline VRK1 & Q99986 & Vaccinia related kinase 1 & 2.84 & 1.28 & 3.65 & 1.03 & 1.67 & 1.72 \\
\hline VRK2 & Q86Y07 & Vaccinia related kinase 2 & 2.27 & 1.00 & 2.27 & $1.54 \%$ & $0.77 *$ & $1.19^{*}$ \\
\hline VRK3 & Q8IV63 & Vaccinia related kinase 3 & 0.67 & 0.98 & 0.65 & $0.77 *$ & 0.82 & 0.63 \\
\hline CAMK2D & Q13557 & $\begin{array}{l}\text { Calcium/calmodulin-dependent protein kinase (CaM kinase) II } \\
\text { delta }\end{array}$ & 0.55 & 0.64 & 0.35 & $0.68 *$ & 0.96 & $0.66^{*}$ \\
\hline GUCY2C & P25092 & Guanylate cyclase $2 \mathrm{C}$ (heat stable enterotoxin receptor) & 0.88 & 0.43 & 0.38 & $0.82 *$ & $0.59 *$ & $0.48^{*}$ \\
\hline PRKCD & Q05655 & Protein kinase $\mathrm{C}$, delta & 0.44 & 0.90 & 0.39 & $0.45^{*}$ & 1.05 & $0.47^{*}$ \\
\hline RPS6KA1 & Q15418 & Ribosomal protein S6 kinase, $90 \mathrm{kDa}$, polypeptide 1 & 0.46 & 0.84 & 0.39 & $0.57 *$ & 1.01 & $0.57^{*}$ \\
\hline TAOK3 & Q9H2K8 & TAO kinase 3 & 0.70 & 0.80 & 0.56 & $0.48^{*}$ & $1.39 *$ & $0.67^{*}$ \\
\hline STK24 & Q9Y6E0 & Serine/threonine kinase 24 (STE20 homolog, yeast) & 0.64 & 1.04 & 0.66 & $0.64 *$ & $1.10^{*}$ & $0.71^{*}$ \\
\hline PDGFRB & P09619 & Platelet-derived growth factor receptor, beta polypeptide & 1.34 & 2.07 & 2.78 & 1.03 & $3.15 *$ & 3.23 \\
\hline BAZ1B & Q9UIG0 & Bromodomain adjacent to zinc finger domain, 1B & 1.17 & 1.47 & 1.72 & $1.22 *$ & $1.41 *$ & $1.72 *$ \\
\hline PRKACB & P22694 & Protein kinase, cAMP-dependent, catalytic, beta & 0.75 & 0.27 & 0.20 & 1.51 & $0.38^{*}$ & 0.57 \\
\hline FRK & P42685 & Fyn-related kinase & 0.21 & 1.15 & 0.24 & 0.49 & $1.89 *$ & 0.93 \\
\hline MST4 & Q9P289 & Serine/threonine-protein kinase MST4 & 1.30 & 1.82 & 2.36 & $0.64^{*}$ & $1.55 *$ & 1.00 \\
\hline
\end{tabular}

Data in italics indicate down-regulated PKs, while data in bold indicate up-regulated PKs with an $\mathrm{FC} \geq 1.5$

$N C$ normal colon, $A D$ adenoma, $A C$ adenocarcinoma

$* q \leq 0.05$, significant differences in quantitative proteomic study [6]

differentiating results of kinase activity were obtained for at least one tissue pair-wise comparison (Table S11). The activity of four PKs was in agreement, while two PKs exhibited an opposite activity when compared to transcriptomic and proteomic directions of their expression. For the protein kinase $\mathrm{C}$ (PKC) delta (PRKCD), six substrates, out of 18 on array, showed differential phosphorylation, two in agreement with expression results and four in opposite direction.

The activity of the one of the selected PKs, STK24, was also analyzed by its immunoprecipitation from pooled tissue samples, followed by phosphorylation in vitro assay (see Electronic supplementary materials for details). As presented in Fig. S3a, the STK24 activity was increased in $\mathrm{AD}$ and $\mathrm{AC}$ tissues, compared with NC. That result was in agreement with immunoblot analyses performed on the same tissue samples, which revealed an increased amount of STK24 kinase in AD and AC (Fig. S3b). However, these results are in contrast to our findings from the large-scale transcriptomic/proteomic surveys.

\section{Discussion}

A variety of proliferative disorders, including cancers, result from deregulated signaling through various PK-mediated pathways, leading to disturbances in cellular homeostasis and uncontrolled cell growth [2]. As inducible PKs are the key elements in phosphorylation-based signal transduction networks, the analysis of particular tumor kinomes may provide valuable information on tumor-specific aberrant signaling. Therefore, the evaluation of tumor kinome profiles may be useful for the selection of PK candidates for targeted anti-cancer therapy.

Genomic analyses of kinome profiles indicate that nearly all human tumors carry at least one mutation in a gene coding for a kinase or phosphatase [4], and several kinase inhibitors selected against altered genes have been shown to possess anti-tumor efficacy in patients with particular genetic alterations. However, the effects of these mutations on gene expression or encoded protein activity may not always be straightforward or predictable. 


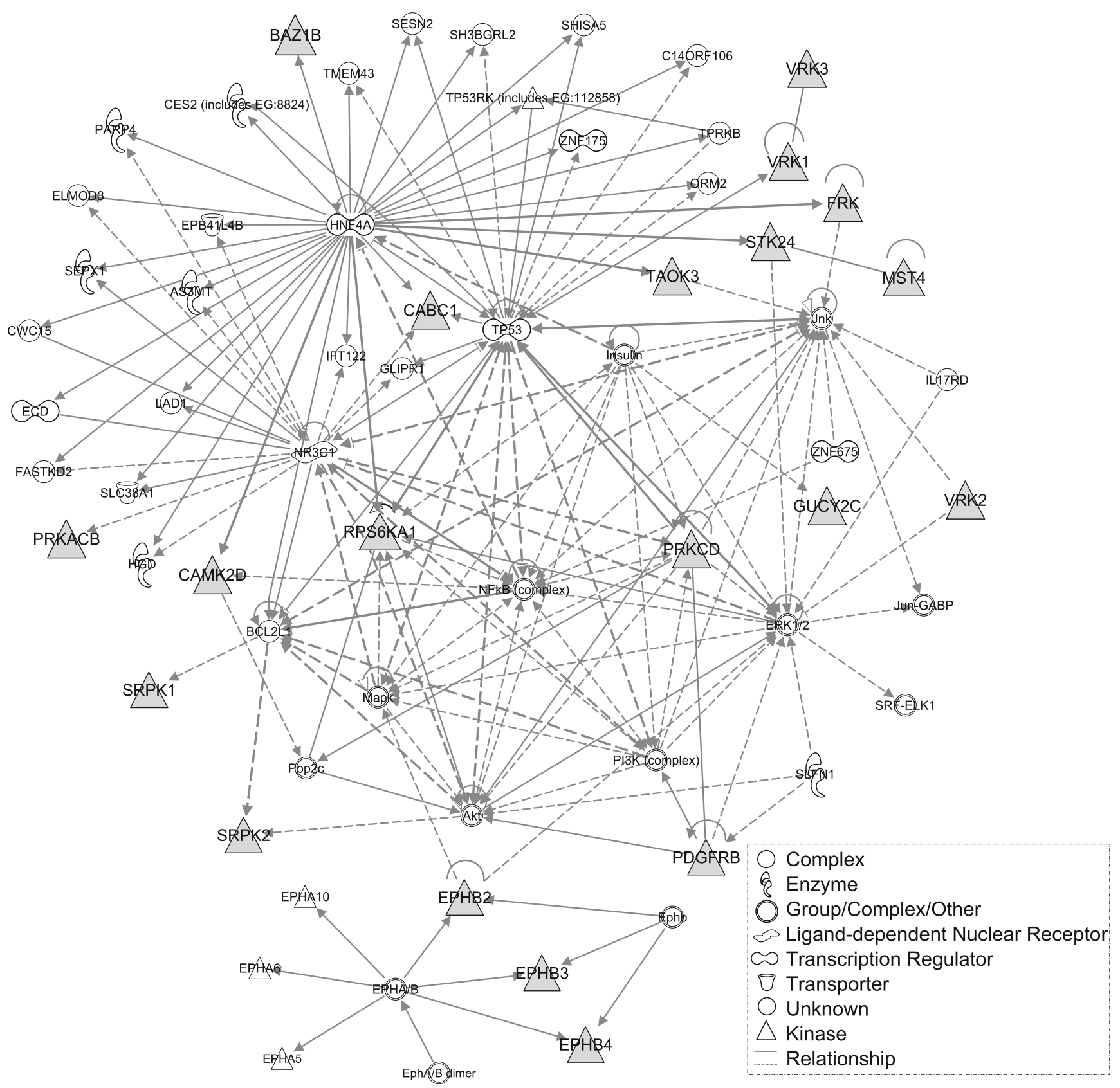

Fig. 2 Merged interaction network of 20 PKs whose expression is differentially regulated during CRC progression. The network was generated by IPA. Gene products are represented as nodes and biological relationships between two nodes as edges. All edges are supported by at least one reference from the literature or from

Our analysis of datasets obtained by integrated transcriptomic and proteomic profiling of CRC tissue samples [6] identified $20 \mathrm{PKs}$ which exhibited concordant changes at the transcriptional and protein levels during CRC progression (Table 2). The expression status of these 20 selected PKs was independently validated by qRT-PCR, in which the direction of the change in expression of 17 genes was consistent with the microarray results. The discrep- canonical information stored in the Ingenuity Pathways Knowledge Base. Node shapes symbolize functional class of the gene product, as specified in the legend. A description of the molecules included in the interaction network is presented in Table S9. The names of the 20 kinases are located over the filled triangles

ancies between the expression results for the remaining three genes may stem from methodological differences inherent in both methods. For example, for the array results for $S R P K 2$, multiple probe sets (203181_x_at; 214931_s_at; 1558254_s_at; 230091_at; 203182_s_at) targeting several gene isoforms were taken into consideration to measure the total mRNA expression. However, the qRTPCR primers used here span a cDNA product that omits the 

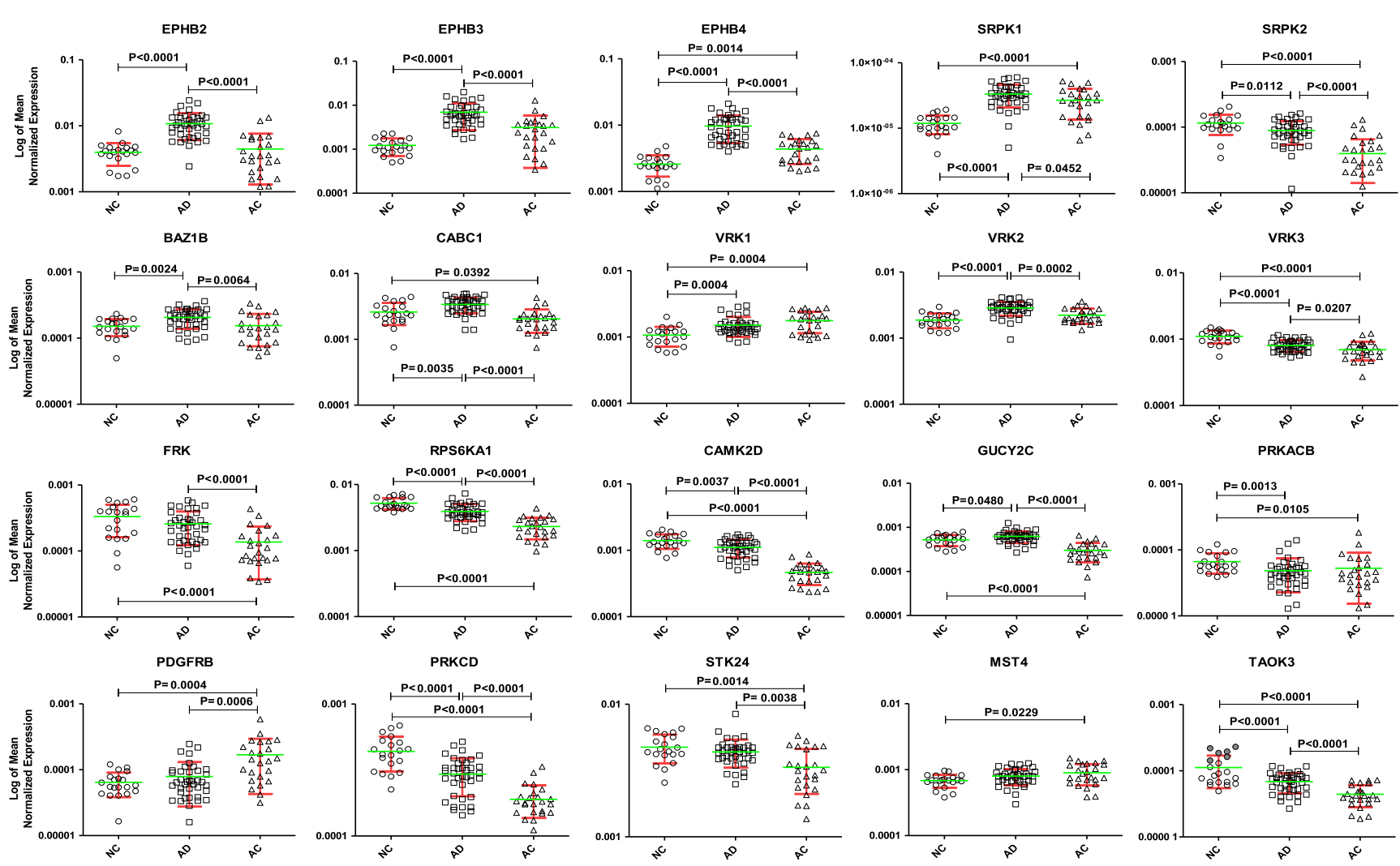

Fig. 3 Analysis of mRNA expression levels of $20 \mathrm{PKs}$ in individual tissue samples. $A C$ adenocarcinoma $(n=24) ; A D$ adenoma $(n=39) ; N C$ normal colon $(n=20)$. One microgram of total RNA was reversetranscribed to generate cDNA and then q-PCR was performed using SYBR Green I chemistry. Data are presented graphically in scatter

isoform targeted by the 214931_s_at probe set. Discrepancies in the reproduction of microarray results by qRT- plots. Green horizontal bars indicate means and red whiskers indicate standard deviation. Differences were analyzed using the MannWhitney test. Open and filled circles in the TAOK3 chart refer to $\mathrm{NC}$ tissue adjacent $(>5 \mathrm{~cm})$ to $\mathrm{AC}(\mathrm{NC}-\mathrm{A})$ and $\mathrm{NC}$ obtained from healthy individuals (NC-D), respectively

PCR were observed previously in ours and others' studies $[7,8]$.
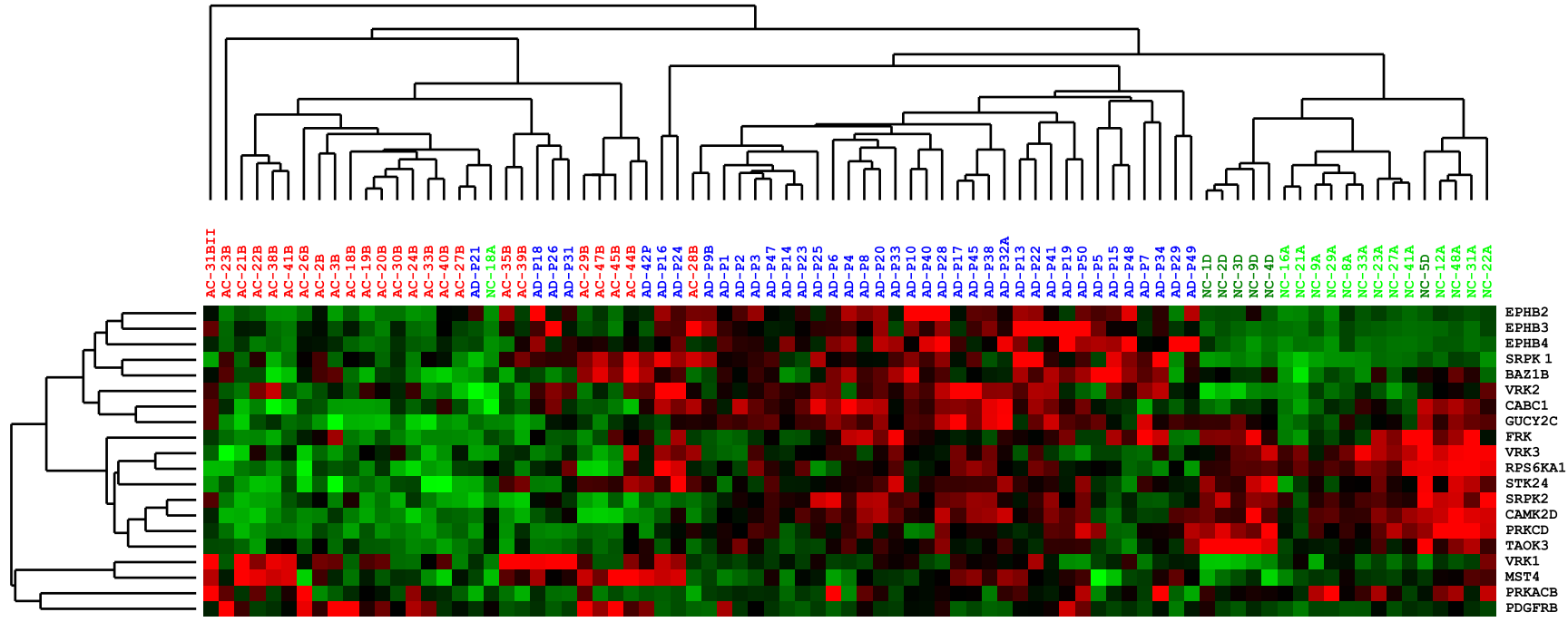

Fig. 4 Hierarchical clustering of PK mRNA expression data. The results from qRT-PCR analysis of 20 PKs (Table S10) were used to generate the cluster tree. Red indicates expression levels greater than the mean, and green indicates expression levels lower than the mean.
$A C$ adenocarcinoma, $A D$ adenoma, $N C-A$ normal colon epithelium adjacent $(>5 \mathrm{~cm})$ to $\mathrm{AC}, N C-D$ normal colon epithelium from healthy individuals 
Most CRCs progress through adenoma to carcinoma as a consequence of altered genetic information. Cancer complexity at the gene level is likely traced to a limited number of altered genetic pathways. Recently, we applied microarray-based gene expression profiles to describe gene regulatory networks, and identified several signaling and metabolic pathways altered in colon tumors [5]. Networks involved in cell proliferation and differentiation, the regulation of gene expression, DNA repair, and cell growth and survival were found to be significantly altered between $\mathrm{NC}$ and colon tumors, while those representing the signaling pathways (TGF-beta, Wnt, PPAR, calcium) or amino acid and lipid metabolism were altered between $\mathrm{AD}$ and $\mathrm{AC}$.

Analysis with the IPA application showed that all 20 selected PKs may be included in one network with the tumor suppressor TP53 as the hub (Fig. 2), suggesting that the kinases differentially expressed during CRC progression are those most commonly associated with the p53 signaling pathway. Interestingly, p53 is considered the predominant alteration in colon cancer, appearing on different levels of molecular interaction networks modeling oncogenic signaling in the colon [5].

Strikingly, three families of PKs were overrepresented among the 20 selected kinases: Eph receptors (three members), vaccinia-related kinases (VRKs; three members, which comprises the entire family), and SRPKs (two members). Hierarchical clustering of mRNAs of 83 tissue samples segregated the selected 20 PK genes into three groups (Fig. 4). The first group represented PKs whose expression was increased during progression to $\mathrm{AD}$ from $\mathrm{NC}$ epithelium and suppressed during malignant transformation of AD to AC. This group included three representatives of the Eph family (EPHB2, EPHB3, and EPHB4), as well as $S R P K 1, B A Z 1 B, V R K 2, C A B C 1$, and GUCY2C. The second cluster contained genes that were down-regulated during CRC progression and included VRK3, CAMK2D, PRKCD, RPS6KA1, TAOK3, STK24, FRK, and SRPK2. The third cluster was more complex and included two upregulated PK genes (VRK1 and MST4); PRKACB, which was down-regulated; and $P D G F R B$, which was overexpressed during malignant transformation of $\mathrm{AD}$ into AC.

Half of the selected PKs were previously described as changed in colorectal tumors or colon cancer cells, including the Eph receptors, SRPK1/2, GUCY2C, PRKCD, RPS6KA1, PRKACB, PDGFRB, and FRK. The PKs most widely reported to be altered in colon cancer are the Eph receptors, which play key roles in the maintenance of cell architecture and in the regulation of cell adhesion and migration (reviewed in [9]). During colorectal tumorigenesis, high expression of crypt EPHB2 and EPHB3 in ADs suppresses tumor progression by a mechanism described as tumor cell compartmentalization, while somatic mutations and promoter hypermethylation in the $E P H B 2$ and $E P H B 4$ genes were identified as potential mechanisms of receptor silencing [10].

Overexpression of SRPK1 has been demonstrated in breast and colonic tumors [11]. Reduced expression of $P R K C D$ was reported in primary human and rat colonic neoplasms, and in several colon AC cell lines [12]. FRK is expressed in epithelial-derived human tissues and cancer cell lines, including breast and colon cancer cells [13]. Guanylyl cyclase C (GUCY2C) maintains homeostasis and genomic integrity in intestinal cells and acts as a tumor suppressor [14]. Amplification of RPS6KA1 was reported in breast and prostate cancers [15], and genetic variation in RPS6KA1 may be associated with CRC risk [16]. PRKACB was implicated in the progression of colon and prostate cancers [17] and platelet-derived growth factor betareceptor (PDGFRB) in lung and colon cancers [18].

The results of the current study show that VRK1/2, MST4, and CAMK2D are aberrantly expressed in colonic tumors. Moreover, this study is the first to report the aberrant expression of TAOK3, STK24, BAZ1B, CABC1, and VRK3 kinases in tumor tissue. VRKs comprise a new STK subfamily with three members [19]. VRK1 protein accumulates in lung carcinomas carrying TP53 mutations [20] and in head and neck squamous cell carcinomas [19], whereas differential expression of VRK2 was demonstrated in estrogen receptor-negative breast cancer [21]. TAOK3, STK24, and MST4 are STE20-related STKs that belong to the germinal center kinase subfamily and are regulated by epidermal growth factor receptor (EGFR) activation [22]. MST4 expression is increased during prostate cancer progression, and TAOK 3 expression is elevated in prostate cancer cells $[22,23]$. Decreased expression of STK24 was shown to enhance the migration potential of breast cancer cells [24]. $\mathrm{Ca}^{2+} /$ calmodulin-dependent PK II (CaMKII) phosphorylates several important proteins in response to increasing intracellular $\mathrm{Ca}^{2+}$ concentration and is involved in the non-canonical $\mathrm{Wnt} / \mathrm{Ca}^{2+}$ signaling pathway. Increased activity of CaMKII has been detected in endometrial [25] and prostate cancer cells in culture [26]; however, little is known about the role of the CaMKII $\delta$ isoform (encoded by $C A M K 2 D$ ) in cancer.

The above-listed PKs are implicated in many aspects of cancer growth. By contrast, to date there are no reports of altered BAZ1B, CABC1, or VRK3 expression in tumors. $B A Z 1 B$ encodes an atypical TK member of the bromodomain protein family, which plays a central role in chromatin-dependent regulation of transcription [27]. $C A B C 1$ (chaperone activity of bc1 complex like) encodes a mitochondrial protein, the expression of which is induced by $\mathrm{p} 53$ in response to DNA damage [28]. CABC1 and VRK3 likely lack phosphorylation activity despite the presence of a PK domain (UniProt database). 
Since changes in mRNA or protein levels do not necessarily result in changes in protein activity, the results should be confirmed by kinome profiling and analysis of kinase activity before conclusions can be drawn regarding kinase function in CRC progression. In fact, we have found rather week concordance between PK activities and the results of large-scale surveys which might be explained by the differences inherent to each of these methods. In addition, although several groups have used peptide arrays for kinome activity analysis [29, 30], this methodology is still in an early stage of development, and important biological and technical challenges remain to be resolved. In a consequence, the differences in phosphorylation levels of potential substrates of a kinase on array (also of opposite direction) has been a quite common phenomenon observed for kinases represented on array by several substrates [29-31].

One of the major caveats to kinome arrays is that they tend to generate false-positive or false-negative results. There are several reasons for this, including: (1) peptide substrates on the array may be exposed to kinases to which they would never be exposed in the cell, (2) consensus peptides can often potentially be phosphorylated by several kinases, (3) consensus sequences on the array may not be the preferred substrate for a given kinase, and (4) peptide substrates may be recognized by the correct kinase but with lower or higher efficiency than in the context of an intact protein. Furthermore, not all potential substrates of a PK are phosphorylated to the same extent [29-31]. We attempted peptide array measurements, but for the reasons mentioned above, the results were insufficient to draw any meaningful conclusions.

The other approach to study kinase activity is in vitro phosphorylation reaction with kinase-specific substrate. However, the data acquired with a monoclonal antibody to determine the activity (Fig. S3a) and amount of STK24 kinase (Fig. S3b) were not consistent with STK24 mRNA obtained by qRT-PCR/transcriptome and protein levels by proteomic datasets (Fig. 3 and Table 2). The inconsistency between immunoblot and proteomic results could be explained by the methodological differences inherent to each of these approaches. For example, quantitative MS analyses allow recognition of multiple peptides derived from a given protein, therefore enabling more reliable protein abundance measurement, while the use of monoclonal antibodies narrows the analysis to specific protein isoforms.

The discrepancies between expression and activity levels of kinase were reported by others previously. Gulmann et al. demonstrated that levels of activated Erk, p38, and JNK kinases were decreased by $25-50 \%$ in CRCs as compared to normal tissue, although total protein levels were similar or even elevated in the cancer tissue samples [32]. Similarly, EGFR expression was reported to be increased in many ACs, including Barrett's carcinoma and Barrett's mucosa [33]; however, its activity is decreased in Barrett's samples when compared with normal squamous epithelium [34]. This discrepancy is likely explained by differential expression/activity of upstream proteins, resulting in differential particular kinase activation, or possibly by the presence of different kinase isoforms, with different mechanisms of activation and/or substrate specificity. In the case of STK24, two isoforms were identified that differentially regulate MAPK pathways as a consequence of differential regulation of these two kinases by protein kinase A (PKA) [35].

To summarize, in addition to the kinases previously described as changed in tumor tissues, we report in the current study the aberrant expression of several kinases in CRC for the first time, including CABC1, BAZ1B, CAMK2D, VRK3, STK24, and TAOK3. These findings may be of diagnostic importance in terms of CRC emergence and the development of novel targeted therapeutics.

Acknowledgments This work was supported by a PBZ-MNiI-2/1/ 2005 grant from the Polish Ministry of Science and Higher Education. Tymon Rubel was supported by the European Union in the framework of the European Social Fund through the Warsaw University of Technology Development Programme.

Conflicts of interest None.

Open Access This article is distributed under the terms of the Creative Commons Attribution Noncommercial License which permits any noncommercial use, distribution, and reproduction in any medium, provided the original author(s) and source are credited.

\section{References}

1. Manning G, Whyte DB, Martinez R, Hunter T, Sudarsanam S (2002) The protein kinase complement of the human genome. Science 298:1912-1934

2. Vogelstein B, Kinzler KW (2004) Cancer genes and the pathways they control. Nat Med 10:789-799

3. Futreal PA, Coin L, Marshall M, Down T, Hubbard T, Wooster R, Rahman N, Stratton MR (2004) A census of human cancer genes. Nat Rev Cancer 4:177-183

4. Greenman C, Stephens P, Smith R, Dalgliesh GL, Hunter C, Bignell G, Davies H, Teague J, Butler A, Stevens C et al (2007) Patterns of somatic mutation in human cancer genomes. Nature 446:153-158

5. Skrzypczak M, Goryca K, Rubel T, Paziewska A, Mikula M, Jarosz D, Pachlewski J, Oledzki J, Ostrowski J (2010) Modeling oncogenic signaling in colon tumors by multidirectional analyses of microarray data directed for maximization of analytical reliability. PLoS One 5:e13091

6. Mikula M, Rubel T, Karczmarski J, Goryca K, Dadlez M, Ostrowski J (2011) Integrating proteomic and transcriptomic high-throughput surveys for search of new biomarkers of colon tumors. Funct Integr Genomics 11:215-224

7. Ostrowski J, Mikula M, Karczmarski J, Rubel T, Wyrwicz LS, Bragoszewski P, Gaj P, Dadlez M, Butruk E, Regula J (2007) Molecular defense mechanisms of Barrett's metaplasia estimated 
by an integrative genomics. Journal of molecular medicine (Berlin, Germany) 85:733-743

8. Grade M, Hormann P, Becker S, Hummon AB, Wangsa D, Varma S, Simon R, Liersch T, Becker H, Difilippantonio MJ et al (2007) Gene expression profiling reveals a massive, aneuploidydependent transcriptional deregulation and distinct differences between lymph node-negative and lymph node-positive colon carcinomas. Cancer Res 67:41-56

9. Arvanitis D, Davy A (2008) Eph/ephrin signaling: networks. Genes Dev 22:416-429

10. Herath NI, Boyd AW (2010) The role of Eph receptors and ephrin ligands in colorectal cancer. Int J Cancer 126:2003-2011

11. Hayes GM, Carrigan PE, Miller LJ (2007) Serine-arginine protein kinase 1 overexpression is associated with tumorigenic imbalance in mitogen-activated protein kinase pathways in breast, colonic, and pancreatic carcinomas. Cancer Res 67:2072-2080

12. Cerda SR, Bissonnette M, Scaglione-Sewell B, Lyons MR, Khare S, Mustafi R, Brasitus TA (2001) PKC-delta inhibits anchoragedependent and -independent growth, enhances differentiation, and increases apoptosis in CaCo-2 cells. Gastroenterology 120:17001712

13. Yim E-K, Siwko S, Lin S-Y (2009) Exploring Rak tyrosine kinase function in breast cancer. Cell Cycle 8:2360-2364

14. Lin JE, Li P, Snook AE, Schulz S, Dasgupta A, Hyslop TM, Gibbons AV, Marszlowicz G, Pitari GM, Waldman SA (2010) The hormone receptor GUCY2C suppresses intestinal tumor formation by inhibiting AKT signaling. Gastroenterology 138:241-254

15. Clark DE, Errington TM, Smith JA, Frierson HF, Weber MJ, Lannigan DA (2005) The serine/threonine protein kinase, p90 ribosomal S6 kinase, is an important regulator of prostate cancer cell proliferation. Cancer Res 65:3108-3116

16. Slattery ML, Lundgreen A, Herrick JS, Wolff RK (2011) Genetic variation in RPS6KA1, RPS6KA2, RPS6KB1, RPS6KB2, and PDK1 and risk of colon or rectal cancer. Mutat Res 706:13-20

17. Merkle D, Hoffmann R (2011) Roles of cAMP and cAMPdependent protein kinase in the progression of prostate cancer: Cross-talk with the androgen receptor. Cell Signal 23:507-515

18. Paulsson J, Sjöblom T, Micke P, Pontén F, Landberg G, Heldin CH, Bergh J, Brennan DJ, Jirström K, Ostman A (2009) Prognostic significance of stromal platelet-derived growth factor betareceptor expression in human breast cancer. Am J Pathol 175:334-341

19. Santos CR, Rodríguez-Pinilla M, Vega FM, Rodríguez-Peralto JL, Blanco S, Sevilla A, Valbuena A, Hernández T, van Wijnen AJ, Li $F$ et al (2006) VRK1 signaling pathway in the context of the proliferation phenotype in head and neck squamous cell carcinoma. Mol Cancer Res 4:177-185

20. Valbuena A, Suarez-Gauthier A, Lopez-Rios F, Lopez-Encuentra A, Blanco S, Fernandez PL, Sanchez-Cespedes M, Lazo PA (2007) Alteration of the VRK1-p53 autoregulatory loop in human lung carcinomas. Lung cancer (Amsterdam, Netherlands) 58:303309

21. Speers C, Tsimelzon A, Sexton K, Herrick AM, Gutierrez C, Culhane A, Quackenbush J, Hilsenbeck S, Chang J, Brown P
(2009) Identification of novel kinase targets for the treatment of estrogen receptor-negative breast cancer. Clin Cancer Res 15:6327-6340

22. Sung V, Luo W, Qian D, Lee I, Jallal B, Gishizky M (2003) The Ste20 kinase MST4 plays a role in prostate cancer progression. Cancer Res 63:3356-3363

23. Romanuik TL, Wang G, Holt RA, Jones SJM, Marra MA, Sadar MD (2009) Identification of novel androgen-responsive genes by sequencing of LongSAGE libraries. BMC Genomics 10:476

24. Ling P, Lu T-J, Yuan C-J, Lai M-D (2008) Biosignaling of mammalian Ste20-related kinases. Cell Signal 20:1237-1247

25. Takai N, Ueda T, Nasu K, Yamashita S, Toyofuku M, Narahara H (2009) Targeting calcium/calmodulin-dependence kinase I and II as a potential anti-proliferation remedy for endometrial carcinomas. Cancer Lett 277:235-243

26. Wang Q, Symes AJ, Kane CA, Freeman A, Nariculam J, Munson P, Thrasivoulou C, Masters JRW, Ahmed A (2010) A novel role for $\mathrm{Wnt} / \mathrm{Ca}^{2+}$ signaling in actin cytoskeleton remodeling and cell motility in prostate cancer. PLoS One 5:e10456

27. Jones MH, Hamana N, Ji N, Shimane M (2000) A novel family of bromodomain genes. Genomics 63:40-45

28. Iiizumi M, Arakawa H, Mori T, Ando A, Nakamura Y (2002) Isolation of a novel gene, $\mathrm{CABC} 1$, encoding a mitochondrial protein that is highly homologous to yeast activity of bc1 complex. Cancer Res 62:1246-1250

29. Diks SH, Kok K, O’Toole T, Hommes DW, van Dijken P, Joore J, Peppelenbosch MP (2004) Kinome profiling for studying lipopolysaccharide signal transduction in human peripheral blood mononuclear cells. J Biol Chem 279:49206-49213

30. Sikkema AH, Diks SH, den Dunnen WFA, ter Elst A, Scherpen FJG, Hoving EW, Ruijtenbeek R, Boender PJ, de Wijn R, Kamps WA et al (2009) Kinome profiling in pediatric brain tumors as a new approach for target discovery. Cancer Res 69:5987-5995

31. de Borst MH, Diks SH, Bolbrinker J, Schellings MW, van Dalen MBA, Peppelenbosch MP, Kreutz R, Pinto YM, Navis G, van Goor H (2007) Profiling of the renal kinome: a novel tool to identify protein kinases involved in angiotensin II-dependent hypertensive renal damage. Am J Physiol Renal Physiol 293:428-437

32. Gulmann C, Sheehan KM, Conroy RM, Wulfkuhle JD, Espina V, Mullarkey MJ, Kay EW, Liotta LA, Petricoin EF 3rd (2009) Quantitative cell signalling analysis reveals down-regulation of MAPK pathway activation in colorectal cancer. J Pathol 218:514-519

33. Jankowski J, Murphy S, Coghill G, Grant A, Wormsley KG, Sanders DS, Kerr M, Hopwood D (1992) Epidermal growth factor receptors in the oesophagus. Gut 33:439-443

34. van Baal JW, Diks SH, Wanders RJ, Rygiel AM, Milano F, Joore J, Bergman JJ, Peppelenbosch MP, Krishnadath KK (2006) Comparison of kinome profiles of Barrett's esophagus with normal squamous esophagus and normal gastric cardia. Cancer Res 66:11605-11612

35. Zhou TH, Ling K, Guo J, Zhou H, Wu YL, Jing Q, Ma L, Pei G (2000) Identification of a human brain-specific isoform of mammalian STE20-like kinase 3 that is regulated by cAMPdependent protein kinase. J Biol Chem 275:2513-2519 